\title{
Turbinmicin inhibits Candida biofilm growth by disrupting fungal vesicle-mediated trafficking
}

\author{
Miao Zhao, ${ }^{1}$ Fan Zhang, ${ }^{2}$ Robert Zarnowski, ${ }^{1}$ Kenneth Barns, ${ }^{2}$ Ryley Jones, ${ }^{1}$ Jen Fossen, ${ }^{1}$ Hiram Sanchez, ${ }^{1}$ Scott R. Rajski, ${ }^{2}$ \\ Anjon Audhya, ${ }^{3}$ Tim S. Bugni, ${ }^{2}$ and David R. Andes' \\ 'Department of Medicine, Medical Microbiology and Immunology, 2Pharmaceutical Sciences Division, and ${ }^{3}$ Department of Biomolecular Chemistry, University of Wisconsin, Madison, Wisconsin, USA. \\ The emergence of drug-resistant fungi has prompted an urgent threat alert from the US Centers for Disease Control (CDC). Biofilm assembly
}

\begin{abstract}
by these pathogens further impairs effective therapy. We recently identified an antifungal, turbinmicin, that inhibits the fungal vesicle-mediated trafficking pathway and demonstrates broad-spectrum activity against planktonically growing fungi. During biofilm growth, vesicles with unique features play a critical role in the delivery of biofilm extracellular matrix components. As these components are largely responsible for the drug resistance associated with biofilm growth, we explored the utility of turbinmicin in the biofilm setting. We found that turbinmicin disrupted extracellular vesicle (EV) delivery during biofilm growth and that this impaired the subsequent assembly of the biofilm matrix. We demonstrated that elimination of the extracellular matrix rendered the drug-resistant biofilm communities susceptible to fungal killing by turbinmicin. Furthermore, the addition of turbinmicin to otherwise ineffective antifungal therapy potentiated the activity of these drugs. The underlying role of vesicles explains this dramatic activity and was supported by phenotype reversal with the addition of exogenous biofilm EVs. This striking capacity to cripple biofilm assembly mechanisms reveals a new approach to eradicating biofilms and sheds light on turbinmicin as a promising anti-biofilm drug.
\end{abstract}

\section{Introduction}

Microorganisms exist predominantly in surface-associated communities called biofilms $(1,2)$. Microbes growing in this state are notorious for their resistance to antimicrobials (2-4). Candida albicans, the most common hospital-acquired fungal infection, frequently forms biofilms on implanted medical devices, leading to lethal disseminated disease (5-7). Treatment recalcitrance is so significant that treatment guidelines recommend the removal of Candida-infected devices $(5,8)$. The intrinsic resistance to therapeutics of these biofilms is multifactorial but largely attributable to the extracellular matrix shielding the biofilm cells (2, 9-11).

We have reported that Candida biofilm extracellular matrix components critical for biofilm cell drug resistance are delivered by extracellular vesicles (EVs) (12-14). The recent discovery of molecules produced by the microbiome of a marine animal identified turbinmicin as an antifungal that targets the vesicle delivery pathway (15). We hypothesized that this therapeutic would subvert the protection afforded by the vesicle-delivered biofilm matrix. Here, we present evidence that turbinmicin inhibited biofilm vesicle production and, in turn, eliminated the extracellular matrix assembly. We found that vesicle reduction rendered biofilm communities susceptible to the antifungal effects of turbinmicin itself, as well as to clinically available antifungal agents. Our findings

Authorship note: $\mathrm{MZ}$ and $\mathrm{FZ}$ contributed equally to this work.

Conflict of interest: The authors have declared that no conflict of interest exists.

Copyright: () 2021, American Society for Clinical Investigation.

Submitted: October 12, 2020; Accepted: December 16, 2020; Published: March 1, 2021.

Reference information: J Clin Invest. 2021;131(5):e145123.

https://doi.org/10.1172/JCl145123. argue that fungal vesicle trafficking pathways represent promising fungal biofilm targets.

\section{Results and Discussion}

Role of turbinmicin in $E V$ delivery of biofilm matrices. We recently identified a broad-spectrum antifungal, turbinmicin (15). The drug exhibits activity against Candida and Aspergillus species during planktonic growth and demonstrates safety and efficacy in animal models of infection. Mode-of-action studies identified Sec14p as the likely target. Sec14p is a phosphatidylinositol/phosphatidylcholine transfer protein required for correct trans-Golgi network dynamics in the vesicle trafficking pathway $(16,17)$.

Extracellular matrix production, a canonical feature of biofilms, is necessary for community fortification and protection from antifungals $(10,18)$. Recent studies demonstrated that EVs deliver much of the Candida biofilm matrix and that this process is critical for drug resistance (13). Given the effect of turbinmicin on fungal vesicle production, we theorized that treatment would subvert vesicle delivery and subsequent matrix assembly, ultimately leaving biofilm cells vulnerable to killing. To test this hypothesis, we initially assessed the impact of turbinmicin on $C$. albicans biofilm vesicle production (Figure 1A). Following exposure to turbinmicin, we observed a more than $500 \%$ reduction in vesicle delivery at the lowest dose tested $(2 \mu \mathrm{g} / \mathrm{mL})$. We found a dose-dependent increase in inhibition with nearly complete abrogation of biofilm vesicles at the highest dose $(16 \mu \mathrm{g} / \mathrm{mL})$. Follow-up studies with 3 additional Candida species (C. tropicalis, C. glabrata, and C. auris) and a strain of Aspergillus fumigatus demonstrated the broad-spectrum impact of this biofilm observation (Supplemental Figure 1, A-D). 
A

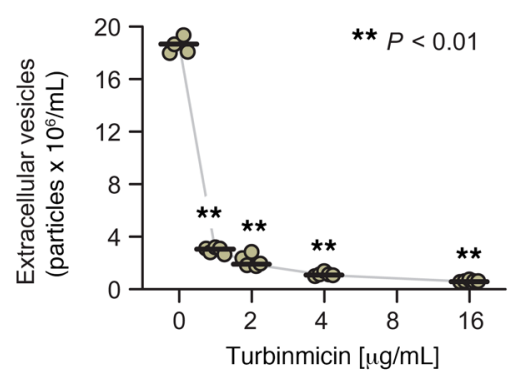

C

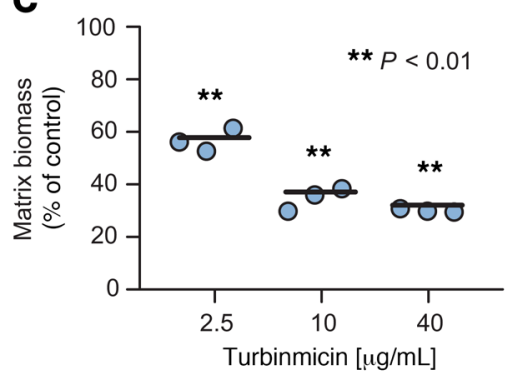

B
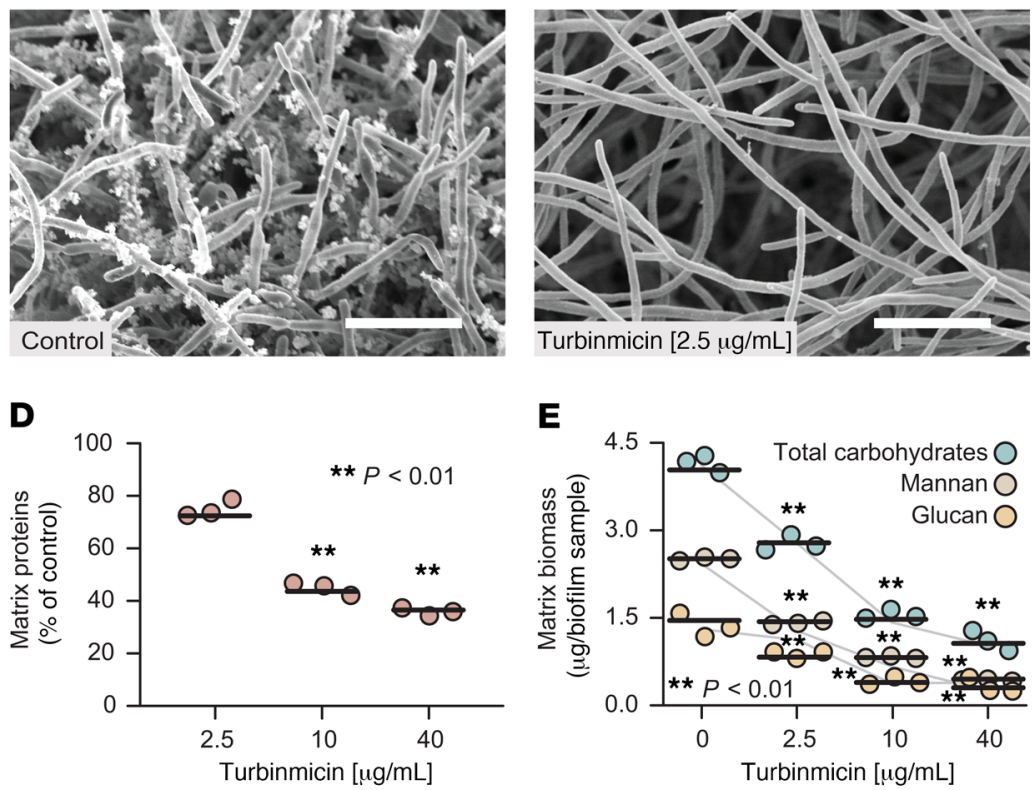

$\mathbf{E}$

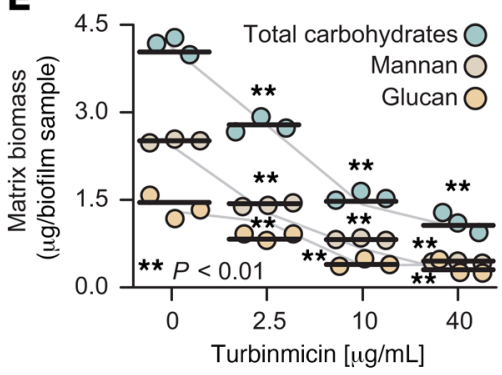

Figure 1. Turbinmicin disrupts biofilm EV matrix delivery. (A) Escalating concentrations of turbinmicin reduced the quantity of $C$. albicans SN250 biofilm EVs from in vitro biofilms over a 24-hour period $(n=5)$ based on imaging flow cytometry. (B) SEM images of $C$. albicans in vitro coverslip biofilms showed a visible reduction of matrix following turbinmicin exposure compared with untreated control samples. Scale bars: $10 \mu \mathrm{m}$. (C) Escalating concentrations of turbinmicin reduced biofilm matrix weight in 6 -well in vitro assays $(n=3)$. Values are expressed as the percentage of the untreated biofilm matrix values. (D) A dose-dependent reduction of biofilm matrix protein in 6-well in vitro assays was observed following exposure to turbinmicin ( $n=3$ ). (E) A dosedependent reduction of biofilm matrix carbohydrate components in 6-well in vitro assays was seen following exposure to turbinmicin ( $n=3$ ). Differences among treatments in $\mathbf{A}$ and $\mathbf{C}-\mathbf{E}$ were assessed by 1-way ANOVA with Tukey's post hoc HSD ( $\left.{ }^{* *} P<0.01\right)$.

Given the role of biofilm vesicles in matrix production, we next performed complementary assays to assess the effect of turbinmicin on the C. albicans biofilm matrix (Figure 1, B-E). Examination of biofilms by scanning electron microscopy (SEM) yielded a striking finding: exposure to turbinmicin $(2.5 \mu \mathrm{g} / \mathrm{mL})$ resulted in a barely visible extracellular matrix compared with control biofilms (Figure 1B). Consistent with our SEM findings, quantitative analyses of biofilm matrices revealed a dose-dependent reduction in biomass upon turbinmicin treatment (Figure 1C). The antifungal protective qualities of the extracellular matrix have been linked to proteins and polysaccharides, including a unique mannan-glucan complex (11). Turbinmicin treatment similarly depleted each of these matrix components, with reductions of nearly $300 \%$ at the highest turbinmicin concentration tested $(40 \mu \mathrm{g} / \mathrm{mL}$; Figure 1, D and E). Our results support a biofilm-relevant action for turbinmicin via eradication of the protective extracellular matrix.

\section{Turbinmicin biofilm efficacy}

We next sought to determine whether turbinmicin is effective against C. albicans biofilms. We propagated biofilms and subsequently treated them with turbinmicin over a 64 -fold concentration range $(0.5-64 \mu \mathrm{g} / \mathrm{mL}$; Figure $2 \mathrm{~A})$. We found that, at between 2 and $4 \mu \mathrm{g} / \mathrm{mL}$, turbinmicin reduced the biofilm burden by $50 \%$ (biofilm minimum inhibitory concentration [MIC]), based on an XTT [(sodium 3'-[1- (phenylaminocarbonyl)- 3,4- tetrazolium]-bis (4-methoxy6-nitro) benzene sulfonic acid hydrate)] assay measurement. This concentration for biofilm inhibition was approximately 4 - to 6 -fold higher than the amount required to inhibit planktonic C. albicans growth (MIC $=0.5 \mu \mathrm{g} / \mathrm{mL}$ ). The need for higher concentrations to inhibit the biofilm over planktonic cells is uniform across available antifungal classes $(3,19)$. However, the MIC ratio (planktonic/biofilm) observed for turbinmicin is lower than that described for other antifungals, which range from 10- to 100-fold for the echinocandins and polyenes to more than 1000 -fold for the triazoles $(2,19,20)$. This highlights the potential of turbinmicin as an anti-biofilm agent. Furthermore, increased turbinmicin concentrations eliminated approximately $80 \%$ of the viable biofilm cells. We also explored the planktonic and biofilm activity of turbinmicin against other fungal species for 3 additional Candida species (C.tropicalis, C.glabrata, and C. auris) and a strain of A. fumigatus. Turbinmicin planktonic MICs for this group were relatively similar: $0.5,0.5,0.125$, and $0.5 \mu \mathrm{g} / \mathrm{mL}$, respectively. In the biofilm assay, turbinmicin likewise exhibited dose-dependent efficacy for these phylogenetically disparate fungal species (Supplemental Figure 2, A-D).

To further elucidate the potential clinical value of turbinmicin biofilm activity, we used a rat central venous catheter model that mimics a severe clinical biofilm infection (Figure 2, B and C, and ref. 21). Following the establishment of biofilms, we instilled turbinmicin for a 24-hour treatment. We then removed catheters and assessed turbinmicin efficacy by measuring the remaining Candida-viable burden and imaging the luminal biofilms. The lower concentration of turbinmicin $(2.5 \mu \mathrm{g} / \mathrm{mL})$ reduced the viable plate counts by nearly $2 \log _{10}$ compared with controls, whereas the higher concentration $(10 \mu \mathrm{g} / \mathrm{mL}$ ) sterilized the catheter (below the detection limit). Using SEM, we observed nearly complete 
A

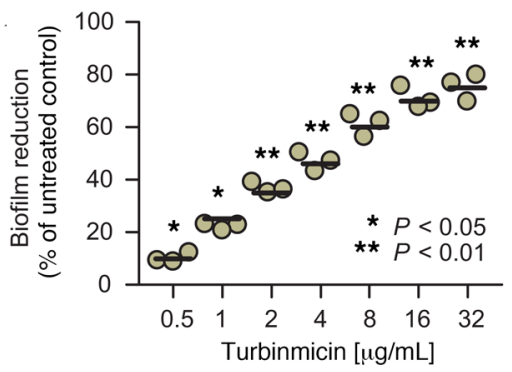

B

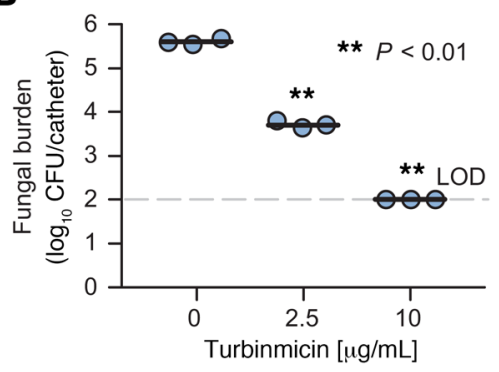

D

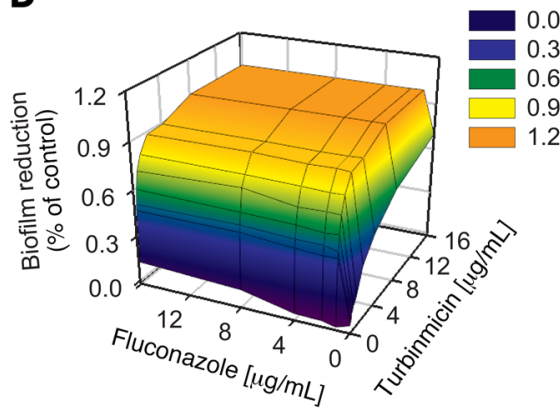

C
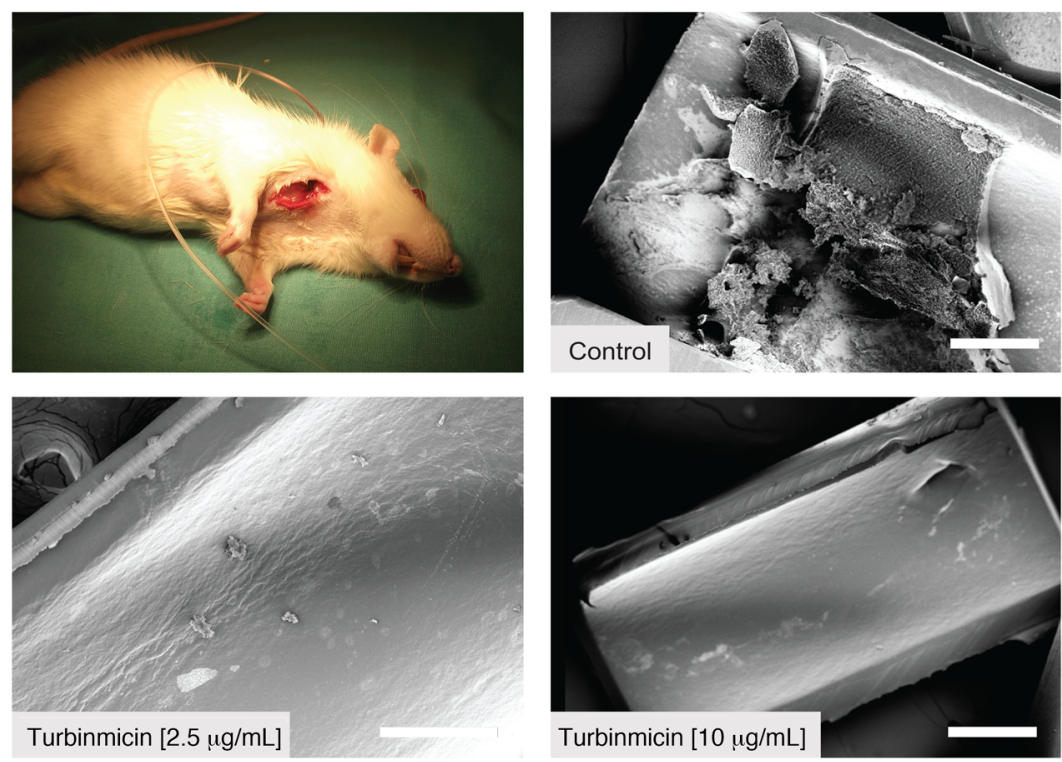

E

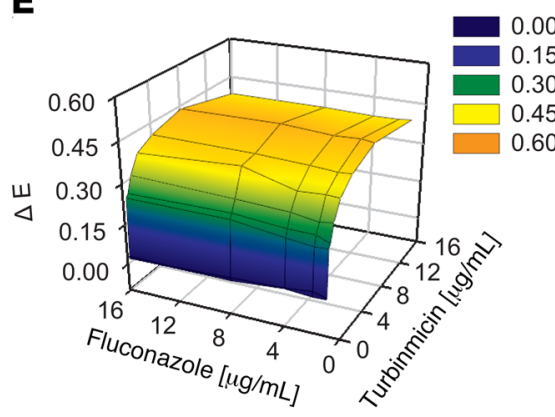

$\mathbf{F}$

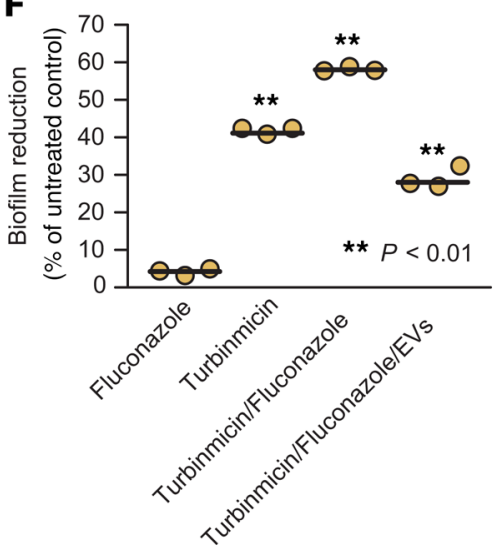

Figure 2. Turbinmicin exhibits vesicle matrix-linked Candida biofilm efficacy alone and in combination with fluconazole. (A) Escalating concentrations of turbinmicin reduced the C. albicans SN250 biofilm burden, as determined by XTT assay using an in vitro biofilm format $(n=3)$. Biofilm activity is expressed as the percentage of biofilm reduction compared with untreated biofilms. (B) Increasing intraluminal doses of turbinmicin eliminated viable C. albicans SN250 in the rat central venous catheter model compared with buffer-treated control animals $(n=3)$. (C) SEM imaging of the intraluminal surface of the rat central venous catheter C. albicans SN250 model demonstrated a visibly reduced biofilm in a dose-response manner follow turbinmicin exposures compared with buffer-treated control animals $(n=3)$. Scale bars: $300 \mu \mathrm{m}$ (D) 3D surface response plot illustrates efficacy enhancement with the combination of turbinmicin and fluconazole compared with monotherapy in a 96-well C. albicans SN250 biofilm assay using an XTT assay ( $n=3$ ). The vertical axis represents the percentage of reduction in biofilm growth compared with untreated control biofilms. Areas in yellow and orange represent enhanced activity due the drug combination. (E) Interaction surfaces obtained from response surface analysis of the Bliss independence drug interaction model for the in vitro combination of turbinmicin and fluconazole against $C$. albicans biofilms. The $x$ and $y$ axes represent the concentrations of turbinmicin and fluconazole, respectively. The $z$ axis is the $\Delta E$. (F) Addition of exogenous vesicles with the antifungal drugs reduced the efficacy of the combination of turbinmicin $(8 \mu \mathrm{g} / \mathrm{mL})$ and fluconazole $(1 \mu \mathrm{g} / \mathrm{mL})$ in a 96 -well C. albicans SN250 biofilm assay using an XTT assay $(n=3)$. Differences among treatments in panels $\mathbf{A}$ and $\mathbf{C}-\mathbf{E}$ were assessed by ANOVA with Tukey's post hoc HSD $\left({ }^{*} P<0.05\right.$ and $\left.{ }^{* *} P<0.01\right)$.

elimination of turbinmicin-treated biofilms, congruent with culture endpoints. These experiments show the utility of turbinmicin as a Candida biofilm therapeutic in a clinically relevant model.

We reasoned that if turbinmicin disrupts the assembly of the protective biofilm matrix, the remaining biofilm cells would be vulnerable to the activity of other antifungals. To test this idea, we elected to examine turbinmicin in conjunction with fluconazole, as Candida biofilms tolerate 1000 -fold greater concentrations of fluconazole than do planktonic cells. We used a checkerboard for- mat to investigate the impact of drug combinations. As expected, monotherapy with turbinmicin showed activity, whereas fluconazole had little effect at the highest concentration. Analyses of the 2 drug combinations revealed enhanced efficacy compared with either drug alone over a wide range of concentrations (these data are depicted in yellow and orange in the surface response plot in Figure 2D). Analysis of the combination of the 2 drugs demonstrated Bliss synergy with $\Delta$ effect $(\Delta E)$ and its $95 \% \mathrm{CI}>0$ (Figure 2, D and E). A large number of the drug combination exposures 
resulted in nearly complete biofilm elimination. We speculate that the enhanced activity of fluconazole in the presence of turbinmicin was due to elimination of the matrix shield that otherwise renders antifungals ineffective.

Previous investigations have found that addition of exogenous EVs to matrix-depleted Candida biofilms can restore matrix function (13). Therefore, to test the theory that the activity of the turbinmicin-fluconazole combination therapy is due to vesicle inhibition of matrix delivery, we performed vesicle add-back experiments (Figure 2F). Remarkably, the addition of EVs to the treated biofilms returned the majority of the community toward the drug-resistant state. The fact that some degree of efficacy was observed despite exogenous vesicles suggests the possibility of an additional, undefined turbinmicin effect. The sum of these findings is consistent with our proposal that turbinmicin biofilm efficacy is linked to the subversion of vesicle matrix delivery. Our observations suggest that EV-based therapeutics may be a useful platform for anti-biofilm strategies and that turbinmicin is a promising broad-spectrum antifungal biofilm agent.

\section{Methods}

Strains and media. C. albicans SN250, C. tropicalis 98-234, C. glabrata 4720, C. auris B11220, A. fumigatus 293 were used. Candida species were sustained on yeast extract-peptone-dextrose (YPD) medium with uridine. A. fumigatus was grown on glucose minimal medium (GMM) $\left(6 \mathrm{~g} /\right.$ liter $\mathrm{NaNO}_{3}, 0.52 \mathrm{~g} /$ liter $\mathrm{KCl}, 0.52 \mathrm{~g} /$ liter $\mathrm{MgSO}_{4} \cdot 7$ $\mathrm{H}_{2} \mathrm{O}, 1.52 \mathrm{~g} /$ liter $\mathrm{KH}_{2} \mathrm{PO}_{4}, 10 \mathrm{~g} /$ liter D-glucose, $15 \mathrm{~g} /$ liter agar supplemented with $1 \mathrm{~mL} /$ liter trace elements). Biofilms were grown in RPMI 1640 buffered with 4-morpholinepropanesulfonic acid (MOPS).

In vitro biofilm models. Three in vitro biofilm models were used, including a 96-well and 6-well polystyrene plate or a glass coverslip. Biofilm drug susceptibility and vesicle production were assessed using the 96-well polystyrene plate assay (22). The 6-well plate assay was used for matrix composition assessment. Biofilm architecture was imaged by SEM of the coverslip.

EV isolation. EVs were isolated from biofilms grown in 6-well polystyrene plates. Media were removed from the plates, filter sterilized, and concentrated using a VivaFlow 200 unit equipped with a Hydrosart $30 \mathrm{kDa}$ cutoff membrane (both from Sartorius). The sample was centrifuged at $10,000 \mathrm{~g}$ for 1 hour at $4^{\circ} \mathrm{C}$ to remove cellular debris. The pellets were discarded, and the supernatant was centrifuged again as described above. This supernatant was then centrifuged at $100,000 \mathrm{~g}$ for 1.5 hours at $4^{\circ} \mathrm{C}$. The supernatants were discarded, and the pellet was resuspended in PBS ( $\mathrm{pH}$ 7.2). Next, the sample was subjected to size exclusion chromatography on a HiPrep 16/60 Sephacryl S-400 HR column (GE Healthcare Life Sciences) pre-equilibrated with PBS (pH 7.2) containing $0.01 \% \mathrm{NaN}_{3}$. All chromatographic separations were performed at room temperature on the HPLC ÄKTApurifier 10 system (Amersham Biosciences).

Quantitative EV analysis. EVs were quantified using a combination of imaging flow cytometry, image confirmation, and fluorescence sensitivity in low-background samples, as previously described (23-25). Prior to analysis, samples were stained with carboxyfluorescein succinimidyl ester (CSFE) and 1,1'-dioctadecyl-3,3,3',3'-tetramethylindocarbocyanine perchlorate (Dil) at $37^{\circ} \mathrm{C}$ for 90 minutes. Excessive dye particles were removed using Illustra Microspin G-50 columns (GE Healthcare). Samples were analyzed on the ImageStreamX Mk II Flow
Cytometry System (Amnis) at $\times 60$ magnification, with default low flow rate/high sensitivity using Inspire software.

In vitro and in vivo biofilm SEM imaging. In vitro biofilms were grown on coverslips in 6-well plates. Ten microliters FCS was placed on each coverslip and dried for 1 hour. Forty microliters of an inoculum of $10^{8}$ cells $/ \mathrm{mL}$ was placed on the coverslip and incubated at $37^{\circ} \mathrm{C}$ for 24 hours. Following incubation, the cells were fixed with $4 \%$ formaldehyde and $1 \%$ glutaraldehyde at $4^{\circ} \mathrm{C}$ overnight. The coverslips were then washed with PBS and treated with $1 \%$ osmium tetroxide for 30 minutes at $22^{\circ} \mathrm{C}$. Samples were subsequently washed with a series of increasing ethanol dilutions (30\% to $100 \%$ ), followed by critical-point drying and coating with platinum. SEM of samples was performed using a LEO 1530 microscope (Zeiss).

In vivo biofilms were propagated in a rat (female, $400 \mathrm{~g}$ Sprague-Dawley) central venous catheter biofilm model as previously described (21). After a 48-hour biofilm formation phase, the devices were removed, sectioned to expose the intraluminal surface, and processed for SEM imaging as described above.

Biofilm matrix isolation and analysis. Biofilms were grown in 6-well plates as described above, and extracellular matrix was collected from mature 48-hour-old biofilms (11). Briefly, biofilms were removed with a spatula in sterile water. Biofilms were then sonicated for 20 minutes, and the matrix was separated from the biomass by centrifugation of the samples at $2880 \mathrm{~g}$ for 20 minutes at $4^{\circ} \mathrm{C}$. To determine the concentration of matrix mannan and glucan, sugars were quantified using a gas-liquid chromatography-flame ionization detector (GLC-FID) on a Shimadzu GC-2010 system after conversion to alditol acetate derivatives (22). A Crossbondä 50\% cyanopropylmethyl $/ 50 \%$ phenylmethyl polysiloxane column was used $(15 \mathrm{~m} \times 0.25 \mathrm{~mm}$ with $0.25 \mu \mathrm{m}$ film thickness; RTX-225, Restek). The GLC conditions were as follows: injector at $220^{\circ} \mathrm{C}$, FID detector at $240^{\circ} \mathrm{C}$, and a temperature program of $215^{\circ} \mathrm{C}$ for 2 minutes, and then $4^{\circ} \mathrm{C} /$ minute up to $230^{\circ} \mathrm{C}$ before holding for 11.25 minutes, run at constant linear velocity of $33.4 \mathrm{~cm} / \mathrm{second}$ and a split ratio of 50:1. Data for these monosugars are presented as micrograms of matrix per milligram of biofilm biomass.

In vitro biofilm and planktonic antifungal susceptibility assay. In vitro biofilm drug susceptibility to microdilution was assessed using a tetrazolium salt XTT reduction assay (22). The percentage of reduction in biofilm growth compared with untreated controls is reported. The CLSI M27 A3 and M38-A2 broth microdilution susceptibility methods were used to determine activity against planktonic Candida and Aspergillus strains, respectively $(26,27)$.

In vivo rat central venous catheter biofilm model. A jugular vein rat catheter infection model was used for in vivo biofilm assessments (Envigo) (21). Quantitative cultures of C. albicans after 24 hours of in vivo growth were utilized to measure viable cell burden. For drug treatment, turbinmicin $(2.5$ or $10 \mu \mathrm{g} / \mathrm{mL})$ was instilled and dwelled in the catheter over a 24 -hour period. The post-treatment viable burden was compared with that of the untreated controls.

Combination therapy analysis. Bliss independence is described by the equation $E_{I N D}=E_{A}+E_{B}-E_{A} \times E_{B}$ for a certain combination of $x \mu \mathrm{g} /$ $\mathrm{mL}$ of drug A and $y \mu \mathrm{g} / \mathrm{mL}$ of drug B. $E_{A}$ is the percentage of biofilm growth inhibition at $x \mu \mathrm{g} / \mathrm{mL}$ of drug A alone, $E_{B}$ is the percentage of biofilm growth inhibition at $y \mu \mathrm{g} / \mathrm{mL}$ of drug B alone, and $E_{I N D}$ is the expected percentage of biofilm growth inhibition of a noninteractive (independent) theoretical combination of $x \mu \mathrm{g} / \mathrm{mL}$ of drug A and $y \mu \mathrm{g} /$ $\mathrm{mL}$ of drug $\mathrm{B}$. The difference $\left(\triangle E=E_{O B S}-E_{I N D}\right)$ between the expected 
percentage of growth inhibition, $E_{I N D}$, and the experimentally observed percentage of growth inhibition, $E_{O B S}$, describes the interaction for each combination of the 2 drugs. If $\Delta E$ and its $95 \%$ CI were greater than $O$ (i.e., $E_{O B S}>E_{I N D}$, and hence more growth inhibition was observed than if the 2 drugs were acting independently), Bliss synergy was concluded for that particular combination. If $\Delta E$ and its $95 \% \mathrm{CI}$ were less than 0 (i.e., $E_{O B S}<E_{I N D}$, and hence less growth inhibition was observed than if the 2 drugs were acting independently), Bliss antagonism was concluded for that particular combination. In any other case where the $95 \% \mathrm{CI}$ of $\Delta E$ would include 0 , the conclusion was Bliss independence $(28,29)$. In each of the independent replicate experiments, the percentage of growth inhibition values for fluconazole and turbinmicin alone were obtained using the Emax model parameters. For each combination of $x \mu \mathrm{g} / \mathrm{mL}$ of fluconazole with $y \mu \mathrm{g} / \mathrm{mL}$ of turbinmicin, the $\triangle E\left(E_{O B S}-E_{I N D}\right)$ was calculated. The interaction was assessed as described above and plotted on a $3 \mathrm{D}$ plot to reveal the interaction surface, with peaks above and below the 0 indicating synergistic and antagonistic combinations, respectively, while the 0 itself indicated no statistically significant interactions.

EV add-back assay. Biofilms were propagated in the wells of 96-well plates. After a 6-hour biofilm formation period, the biofilms were washed twice with PBS. Biofilms were then treated with either turbinmicin $(8 \mu \mathrm{g} / \mathrm{mL})$, fluconazole $(1 \mu \mathrm{g} / \mathrm{mL})$, or the combination of turbinmicin and fluconazole. Exogenous purified EVs collected from 48-hour biofilms were administered at a concentration of 21,804 \pm $1711 \mathrm{EVs} / \mathrm{mL} 1$ hour before antifungal therapy for 1 series of wells. Following another 24 hours of incubation, the quantity of Candida bio- film cells was assessed by XTT assay. The results are presented as a percentage of reduction by comparing untreated biofilms with those that were treated.

Statistics. Data sets were analyzed by 1-way ANOVA with Tukey's post hoc honestly significant difference (HSD) test.

Study approval. Animal procedures were approved by the IACUC of the University of Wisconsin (protocol DA0031).

\section{Author contributions}

TSB, DRA, and AA conceptualized the study. FZ, MZ, RZ, JF, HS, and $\mathrm{KB}$ developed the study methodology. FZ, MZ, FZ, RZ, HS, and AA conducted formal analysis. FZ, MZ, RZ, HS, JF, and RJ performed experiments. MZ, FZ, RZ, JF, HS, SRR, AA, DRA, and TSB wrote, reviewed, and edited the manuscript. TSB and DRA supervised the study. DRA and TSB acquired funding.

\section{Acknowledgments}

This study was funded by the National Institute of Allergy and Infectious Diseases (NIAID), NIH (R01AI073289 and U19AI142720).

Address correspondence to: David Andes, Departments of Medicine and Medical Microbiology and Immunology, University of Wisconsin-Madison, 1685 Highland Ave., Madison, Wisconsin 53705, USA. Phone: 608.220.9622; Email: dra@medicine.wisc. edu. Or to: Tim Bugni, 6111 Rennebohm Hall, 777 Highland Ave, Madison, WI 53705-2222, USA. Phone: 801.440.7123; Email: Tim.bugni@wisc.edu.
1. Costerton JW, et al. Bacterial biofilms: a common cause of persistent infections. Science. 1999;284(5418):1318-1322.

2. Chandra J, et al. Biofilm formation by the fungal pathogen Candida albicans: development, architecture, and drug resistance. JBacteriol. 2001;183(18):5385-5394.

3. Taff HT, et al. Mechanisms of Candida biofilm drug resistance. Future Microbiol. 2013;8(10):1325-1337.

4. Desai JV, et al. Fungal biofilms, drug resistance, and recurrent infection. Cold Spring Harb Perspect Med. 2014;4(10):a019729.

5. Pappas PG, et al. Clinical practice guideline for the management of candidiasis: 2016 update by the Infectious Diseases Society of America. Clin Infect Dis. 2016;62(4):e1-e50.

6. Kojic EM, Darouiche RO. Candida infections of medical devices. Clin Microbiol Rev. 2004;17(2):255-267.

7. Pfaller MA, Diekema DJ. Epidemiology of invasive candidiasis: a persistent public health problem. Clin Microbiol Rev. 2007;20(1):133-163.

8. Andes DR, et al. Impact of treatment strategy on outcomes in patients with candidemia and other forms of invasive candidiasis: a patient-level quantitative review of randomized trials. Clin Infect Dis. 2012;54(8):1110-1122.

9. Ramage G, et al. Candida biofilms on implanted biomaterials: a clinically significant problem. FEMS Yeast Res. 2006;6(7):979-986.

10. Taff HT, et al. A Candida biofilm-induced pathway for matrix glucan delivery: impli- cations for drug resistance. PLoS Pathog. 2012;8(8):e1002848.

11. Mitchell KF, et al. Community participation in biofilm matrix assembly and function. Proc Natl Acad Sci U S A. 2015;112(13):4092-4097.

12. Zarnowski R, et al. Novel entries in a fungal biofilm matrix encyclopedia. $m$ Bio. 2014;5(4):e01333-e01314.

13. Zarnowski R, et al. Candida albicans biofilm-induced vesicles confer drug resistance through matrix biogenesis. PLoS Biol. 2018;16(10):e2006872.

14. Dominguez E, et al. Conservation and divergence in the Candida species biofilm matrix mannan-glucan complex structure, function, and genetic control. mBio. 2018;9(2):e00451-18.

15. Zhang $\mathrm{F}$, et al. A marine microbiome antifungal targets urgent-threat drug-resistant fungi. Science. 2020;370(6519):974-978.

16. Bankaitis VA, et al. An essential role for a phospholipid transfer protein in yeast Golgi function. Nature. 1990;347(6293):561-562.

17. Curwin AJ, et al. Phospholipid transfer protein Sec14 is required for trafficking from endosomes and regulates distinct trans-Golgi export pathways. J Biol Chem. 2009;284(11):7364-7375.

18. Flemming HC, Wingender J. The biofilm matrix. Nat Rev Microbiol. 2010;8(9):623-633.

19. Ramage $\mathrm{G}$, et al. Biofilms of Candida albicans and their associated resistance to antifungal agents. Am Clin Lab. 2001;20(7):42-44.

20. Nett JE, et al. Role of Fks1p and matrix glucan in Candida albicans biofilm resistance to an echi- nocandin, pyrimidine, and polyene. Antimicrob Agents Chemother. 2010;54(8):3505-3508.

21. Andes D, et al. Development and characterization of an in vivo central venous catheter Candida albicans biofilm model. Infect Immun 2004;72(10):6023-6031.

22. Ramage G, et al. Standardized method for in vitro antifungal susceptibility testing of Candida albicans biofilms. Antimicrob Agents Chemother. 2001;45(9):2475-2479.

23. Zaborowski MP, et al. Extracellular vesicles: composition, biological relevance, and methods of study. Bioscience. 2015;65(8):783-797.

24. Headland SE, et al. Cutting-edge analysis of extracellular microparticles using ImageStream(X) imaging flow cytometry. Sci Rep. 2014;4:5237.

25. Lannigan J, Erdbruegger U. Imaging flow cytometry for the characterization of extracellular vesicles. Methods. 2017;112:55-67.

26. Institute CLS. Reference Method For Broth Microdilution Antifungal Susceptibility Testing Of Yeasts, 3rd ed. Clinical Laboratory Standards Institute; 2007.

27. Institute CLS. Reference Method For Broth Dilution Antifungal Susceptibility Testingof Filamentous Fungi, 3rd ed. Clinical Laboratory Standards Institute; 2017.

28. Bliss CI. The calculation of microbial assays. Bacteriol Rev. 1956;20(4):243-258.

29. Liu Q, et al. Evaluation of drug combination effect using a Bliss independence doseresponse surface model. Stat Biopharm Res. 2018;10(2):112-122. 